\title{
8 Philosophische Anthropologie als Rahmen für die heutige Hirn- und Verhaltensforschung: Zwischen Mitmachen, Nachmachen und Nachahmen
}

Während der beiden letzten Jahrzehnte hat es bedeutende Aufschwünge sowohl in der neurobiologischen Hirnforschung als auch in der vergleichenden Verhaltensforschung gegeben. Ich beginne mit einer Erläuterung dieser Fortschritte, zunächst in der Hirnforschung, die das Gehirn als selbstreferentielles System modelliert und zugleich die sog. Spiegelneuronen entdeckt hat, sodann in der Verhaltensforschung, näher in der vergleichenden Primatologie. Im dritten Punkt führe ich die Philosophische Anthropologie als einen Rahmen ein, in dem man die Probleme der Hirnforschung und der Verhaltensforschung aufeinander beziehen kann, was bisher aus diesen beiden Forschungsrichtungen aufeinander nicht geschieht. Im vierten und letzten Schritt verdeutliche ich diese Rahmenfunktion der Philosophischen Anthropologie exemplarisch an der grundlegenden Unterscheidung zwischen dem aktualen Mitmachen, dem zeitlich versetzten Nachmachen und der Nachahmung personalen Verhaltens (imitation statt mimicry). Diese von Helmuth Plessner in seiner Philosophischen Anthropologie begründete Differenzierung ist erneut aktuell geworden, da für ihre Relata Korrelate in der Hirn- und Verhaltensforschung entdeckt werden konnten, ohne dass die Kausalbeziehungen bereits klar geworden wären. Da aber weder die neurobiologische Hirnforschung noch die vergleichende Primatologie über eine Rahmentheorie verfügen, in der sie sich selbst im Zusammenhang mit der jeweils anderen Forschungsrichtung verstehen können, fallen einem die Beziehungen zwischen den verschiedenen Korrelata und Relata erst in ihrem gemeinsamen Rahmen der Philosophischen Anthropologie auf. Diese Einordnung neuer Erkenntnisse hilft, nicht nur übereilte, sondern vor allem überverallgemeinerte Erkenntnisansprüche, die in den letzten Jahren teils aus der Hirnforschung und teils aus der Primatologie erhoben worden sind, $\mathrm{zu}$ begrenzen, wodurch die Grundlagenforschung fortgesetzt werden kann. 


\subsection{Zur Hirnforschung: Metarepräsentationen oder die Selbstreferenz des Gehirns und die Repräsentation der Spiegelneuronen}

Die neurobiologische Hirnforschung hat sich von dem alten Modell, das Gehirn funktioniere wie ein Computer, verabschiedet. Es gebe im Gehirn keine Trennung zwischen Hardware und Software, so lautet die Auskunft der meisten führenden Neurobiologen, denkt man z. B. an die Nobelpreisträger Eric Kandel und Gerald M. Edelman in den USA, an Wolf Singer in Frankfurt am Main und an Gerhard Roth in Bremen. Man müsse das sog. Bindungsproblem anders lösen. Dieses Problem besteht in der folgenden Frage: Wie kommt es zur Einheitlichkeit des Verhaltens eines Lebewesens in der Umwelt? Insoweit an der Einheitlichkeit des Verhaltens Bewusstsein beteiligt ist, lautet die Frage auch: Wie kommt es zur Einheitlichkeit der Wahrnehmung, so dass das Lebewesen mit einer Verhaltenseinheit auf die wahrgenommene Situation antworten kann? Negativ formuliert: Warum zerfällt das Verhalten, das man von außen beobachten kann, nicht in lauter Einzelteile? Warum zerfällt schon die Wahrnehmung nicht in viele Einzelteile, wodurch die Antwortreaktion auf sie paralysiert wäre? Die Neurobiologen suchen nach neuronalen Korrelaten für das, was sie von außen als Verhaltenseinheit beobachten können, nehmen also an, dass es im Gehirn ein Korrelat für die Einheitlichkeit des Verhaltens und der Wahrnehmung von außen gebe. Wenn es ein Korrelat für diese Einheit gibt, dann müsste es innerhalb des Gehirns zu einer Verbindung verschiedener neuronaler Aktivitäten kommen, nämlich der Aktivitäten von einzelnen Neuronen über Gruppen von Neuronen in verschiedenen Arealen des Gehirns bis zur Verbindung dieser Gruppen-Aktivitäten in mehreren Arealen zugleich (Synchronisation). Durch die neuen bildgebenden Methoden (die Computertomographie, die Magnetresonanztomographie) kann man die Korrelate indirekt und mit geringem zeitlichen Abstand visualisieren. Aber diese Bilder müssen interpretiert werden: Man muss etwas in sie hinein- und aus ihnen herauslesen können. Diese Bilder werden auch nicht vollständig ersetzt, sondern ergänzt von den alten Ableitungen aus Elektroden, die man implantiert, um Elektropotentiale direkt messen zu können. Für beides, die Bilder und Messungen, braucht man Modelle. Was tritt an die Stelle des Computers?

Es gibt eine ganze Palette von Modellen. An dem einen Ende geht man von Lebewesen aus, die gewiss Triebe wie Säugetiere haben, die sie befriedigen möchten, z. B. Hunger und Durst. Man baut die Experimente so in diese Triebbefriedigungen ein, dass die Tiere kooperieren. Je höher diese Tiere entwickelt sind, desto schwieriger wird dies. Primaten brauchen oft ein halbes Jahr an 
Trainingszeit, bis sie die Versuchsdesigns für Wahrnehmungsexperimente kontrolliert erlernt haben. Es stellt sich dann die Frage, wie viel menschliches Telos in die Experimentalsituationen bereits eingebaut wurde, da sie von den Versuchstieren erst aufwendig erlernt werden mussten. Inwiefern haben die Probanden dies auf die gleiche Weise wie Menschen erlernt, oder auf eine davon verschiedene Weise?

Auf der anderen Seite des Spektrums der Modelle liegen Reflexionsmodelle, z. B. Singers „Beobachter im Gehirn“, die von vornherein damit rechnen, dass die Probanden mehr als primitive Triebbefriediger sein können. Hier geht es um die Modellierung der sog. höheren Funktionen des Gehirns und Bewusstseins. Die Grundidee ist die folgende: Man muss über die Erfassung von neuronalen Repräsentationen hinausgehen zu der Darstellung von Metarepräsentationen. Dieser Weg führt von der Repräsentation von etwas Äußerem im Inneren des Gehirnes $z u$ der Repräsentation dieses Inneren im Gehirn durch Repräsentation im Inneren des Gehirns (Singer 2002, 70-72), oder in anderer Terminologie: von der Repräsentation des Äußeren (Stimulus, Objekts) zur Repräsentation des Inneren im Gehirn durch Repräsentation im Inneren des Gehirns (zum „Reichtum der inneren Repräsentationen“, die im Unterschied zu den äußeren Repräsentationen des alten Behaviorismus im Gehirn selbst „zwischen Reizen und Reaktionen vermitteln“ (Kandel 2008, 203). Dieser Übergang von der Repräsentation des Äußeren im Gehirn zu der Repräsentation der bereits dem Gehirn internen Repräsentation nochmals im Inneren des Gehirns, also der Metarepräsentation, ist umso wichtiger, je höher die Versuchstiere entwickelt sind, je näher sie dem Menschen kommen.

Als eine Repräsentation galt früher ein neuronales Korrelat für die im Äußeren beobachtete Verhaltenseinheit, aber dieses Korrelat in der Form neuronaler Aktivitäten innerhalb des Gehirns kann man nun methodisch darstellen. Das neuronale Korrelat einer Repräsentation liegt zeitlich zwischen dem sensorischen Eingang und dem motorischen Ausgang beim Versuchstier. Pawlows Hund ist ein Beispiel dafür, dass er einen bedingten Reflex erlernen kann, d. $h$. eine Repräsentation durch Konditionierung. Noch primitiver ist eine instinktive, d. h. nicht erlernte, sondern angeborene Repräsentation. Dieses Dreieck aus sensorischem Input, neuronalem Korrelat und motorischem Output ist repräsentativ, insoweit es unter gleichen Bedingungen mit hoher Wahrscheinlichkeit reproduziert werden kann. Die Gründe dafür können ganz verschiedene sein, von angeborener Verschaltung bis zu erlernter Verschaltung der Sensorik mit der Motorik im Gehirn.

Aber nach diesem alten, behavioristischen Muster der Repräsentation (statt Metarepräsentation: Singer, oder der äußeren statt inneren Repräsentation: 
Kandel) kann keine Intelligenz funktionieren. Es ist keine Intelligenz vorhanden, wenn man angeboren, d. h. instinktiv, oder durch bedingten Reflex oder durch bloße Induktion nach einer Serie von Trial and Error reagiert. Ein Menschenaffe handelt in dem Maße intelligent, in dem er durch plötzliche Einsicht ein Verhaltensproblem löst, das er bisher nicht lösen konnte (Scheler 1995, 32f.). Er verwendet etwas als Werkzeug, das bisher kein Werkzeug war, stapelt Kisten aufeinander, um an eine entfernte Frucht zu gelangen, oder schenkt einem Artgenossen einen Vorteil, um später einen anderen Vorteil zurückzubekommen. Das intelligente Verhalten geht nicht in der schnellen motorischen Antwort auf eine sensorische Situation hier und jetzt auf, sondern schaltet Vermittlungsglieder und Umwege ein. Es nimmt Abstand von der aktuellen Wahrnehmungssituation, um viele Situationen im Vergleich der Vorstellungen und Einbildungen durchlaufen $\mathrm{zu}$ können, bis es zur überraschenden Antwort kommt. Intelligentes Verhalten dauert nicht nur länger als die schnelle Wiederholung eines Standardexperiments, die nur den senso-motorischen Habitus zu überprüfen vermag, nicht aber Intelligenz.

Intelligentes Verhalten übergreift mehrere Situationen der Wahrnehmung und Vorstellung, ehe es sich die Lösung des Verhaltensproblems imaginieren und ausprobieren kann. Das Verhalten wird vor allem stärker abhängig von den Gedächtniskapazitäten, durch die verschiedene Situationen miteinander verglichen werden können. Da die Einheit der Wahrnehmung erst durch die Gedächtnisvergleiche hindurch gebunden wird, hat Roth das Gedächtnis „unser wichtigstes ,Sinnesorgan'“ genannt (Roth 1997, 263). Edelman ersetzt sogar die Redeweise von Repräsentationen durch die des „re-entry“, d. h. durch den Wiedereintritt der Aktivität von neuronalen Schaltkreisen in die Aktivität von neuronalen Schaltkreisen:

\footnotetext{
But now there is no signal from the original object. Instead there is stimulation, within the brain of a subject, of reentrant circuits to yield an image or thought of the object upon memory recall. In this case, the image is brought up by means of the brain speaking to itself. (Edelman 2006, 32)
}

Es ist also viel schwieriger, für intelligentes Verhalten neuronale Korrelate $\mathrm{zu}$ finden, als für angeborenes Verhalten oder für erlernte bedingte Reflexe. Selbst assoziatives Lernen kann man leicht nach der Wahrscheinlichkeit durch Wiederholung der sensorischen, neuronalen und motorischen Komponenten bestimmter Situationen modellieren. Aber intelligentes Verhalten wird vermittelter statt unmittelbar, indirekter statt direkt gebildet. Seine Korrelate durchlaufen alle möglichen Areale des Gehirns in immer neuen Feedback-Schleifen. Die neuronalen Aktivitätsmuster werden nicht nur räumlich immer komplexer, sie 
werden vor allem eine Frage der zeitlichen Synchronisation. Wenn es gar keine umkehrbar eindeutige Zuordnung zwischen der sensorischen Situation und der motorischen Antwort hier und jetzt gibt, dann treten anstelle der Repräsentationen die Metarepräsentationen oder eben ein reentry als neuronale Korrelate hervor. Die räumliche und zeitliche Verteilung der Repräsentationen im Gehirn allein hier und jetzt reicht nicht aus, um darin das Korrelat für die Einheit eines intelligenten Verhaltens darstellen zu können, das viele Situationen der Wahrnehmung übergreift. Es fehlt an Langzeitstudien, die offene Lernsituationen betreffen.

Man kann sich die Bedeutung der Metarepräsentation auch schon rein quantitativ an den Proportionen der neuronalen Verbindungen in unserem Gehirn verdeutlichen: Ein menschliches Gehirn enthalte ca. $10^{14}$ Verbindungen zwischen den Neuronen. Davon stelle etwa jede zehnmillionste Verbindung eine Außenverbindung unseres Körpers durch seine Sensorik und Motorik her. Alle anderen Verbindungen dienen nur dem Informationsaustausch zwischen den Neuronen des Gehirns innerhalb des Gehirns ohne Außenverbindungen. Das Verhältnis zwischen den Innenverbindungen und den Außenverbindungen ist also das Verhältnis von $10^{7} \mathrm{zu}$. Auf eine einzige Außenverbindung kommen 10 Millionen Innenverbindungen (Spitzer 2002, 52). Selbst wenn man nicht die neuronalen Verbindungen betrachtet, sondern nur das Verhältnis zwischen den funktionalen Ortungen im Kortex nach Volumina schätzt, dann ergibt sich ein Verhältnis zwischen den Kortex-Volumina, in denen man Außenbezüge (Wahrnehmung und Motorik), und den Kortex-Volumina, in denen man internen Informationsaustausch lokalisiert. Bei Ratten, die sehr häufig als Versuchstiere verwendet werden, betrage dieses Verhältnis zwischen funktionalem Außen und Innen $90 \mathrm{zu}$ 10, obgleich wir ca. $90 \%$ der Gene mit ihnen teilen. Demgegenüber betrage es bei Menschen umgekehrt $10 \mathrm{zu} 90$ (vgl. Storch/Welsch/ Wink 2001, 375).

Es ist in Anbetracht solcher quantitativer Relationen kein Wunder, dass Neurobiologen wie Wolf Singer oder Gerald Edelman angesichts dieser Komplexität des menschlichen Gehirns Reflexionsmodelle für das Gehirn entwickeln: Das Gehirn wird hermeneutisch so modelliert, als ob es sich in sich selbst beobachten oder mit sich sprechen könnte, indem neuronale Aktivitäten aus verschiedenen Arealen des Gehirns über verschiedene und parallel laufende Feedback-Schleifen in der Zeit synchronisiert werden. Die Repräsentationen werden in n-dimensionalen Proportionen durch Metarepräsentationen oder reentries kontextualisiert. Die aktualen Repräsentationen führen nicht von sich aus direkt zu motorischen Antworten, sondern werden in die Selbstreferenz des Gehirnes eingeordnet. Erst in dem Maße, in dem es Abweichungen von den Nor- 
malwerten im Hintergrundrauschen des Gehirnes gibt, wird die Aufmerkung eingeschaltet, um Referenz auf Äußeres innerhalb der Selbstreferenz des Gehirns zu ermöglichen.

Wenn man die Neurobiologie nicht immer nur auf Rattenniveau durchführen will, dann braucht man solche anspruchsvollen Reflexionsmodelle, um die neuronalen Korrelate für das unbewusste, emotionale und kognitive Selbstbewusstsein erforschen zu können. Wer auf dem Niveau von Ratten verbleibt, sollte sich nicht ständig bestimmter Fehlübertragungen auf Menschen und andere Primaten schuldig machen, eben Übertragungen, die bereits quantitativ der Anzahl und Dimension der jeweiligen neuronalen Verbindungen widersprechen. Diese Übertragung ist einfach faktisch falsch, da die quantitativen Unterschiede auch in systemisch verschiedene Qualitäten umschlagen können. Bekanntlich kann schon systemisch gesehen ein und dieselbe Funktion durch verschiedene Strukturen ausgeübt werden. Wir müssen in der Grundlagenforschung, abgesehen von ganz elementaren und lokalisierbaren Funktionen in der Sensorik und Motorik, umgekehrt fragen: Wenn auch Rattenhirne nicht ohne die wenigstens 10 Prozent intern verbundener Kortex-Volumina funktionieren, gehören dann nicht Metarepräsentationen schon quantitativ betrachtet zur Funktionsweise selbst einfacher Gehirne?

Das ganze Repräsentationsmodell muss vom Standpunkt der Metarepräsentationen oder reentries, die vor allem durch Synchronisation zustande kommen, in Frage gestellt werden. - Ich habe diesen Durchbruch der neurobiologischen Hirnforschung zur selbstreferentiellen Funktionsweise des Gehirns gegen den alten Behaviorismus und Empirismus schon oft gewürdigt und unterstützt, aber dabei zugleich hervorgehoben, dass die Selbstreferenz des Gehirns nur neurophysisches Korrelat und keineswegs das wirkliche Substitut für die Selbstreferenz des Bewusstseins, der Sprache und anderer Symbolarten sein kann (siehe Krüger 2004a; 2007a; 2010). Erst dank der bleibenden Selbstreferenz von Bewusstsein und Symbolen im personalen Verhalten, die von anderer Qualität als der des Korrelates sind, konnten die Modelle, das Gehirn in seiner selbstreferentiellen Funktionsweise $\mathrm{zu}$ verstehen, entwickelt werden. Niemand ist experimentell dazu in der Lage, aus der Selbstreferenz des Gehirns die des Bewusstseins und die der Sprache wirklich herzustellen oder abzuleiten.

Max Scheler und Helmuth Plessner haben in ihren Philosophischen Anthropologien nicht mit dem Modell der Repräsentation von Äußerem gearbeitet und brauchten daher auch nicht das Vokabular der Metarepräsentationen oder der reentries, um die Grenzen dieses Repräsentationsmodells aufzuzeigen. Beide haben klar gesagt, dass sich nicht einmal intelligentes, umso weniger geistiges Verhalten durch bloße Assoziation im Sinne der Wahrscheinlichkeit von 
Versuch und Irrtum erklären lassen. Scheler und Plessner haben auf einer Dissoziation zunächst der Instinkte (angeborenes Verhalten), sodann aber auch habituierter Assoziation bestanden, um Korrelate für dieses höhere Verhalten suchen zu können. Die instinktive Verknüpfung von Sensorik und Motorik müsse erst dissoziiert werden, damit ein Korrelat für höheres Verhalten entstehen könne. Für Scheler waren das Heraustreten relativer Einzelempfindungen, ihre assoziative Verknüpfung und die Intelligenz nicht drei Evolutionsphasen nacheinander, sondern drei „gleich ursprüngliche Entwicklungsprodukte (Zerfallsprodukte - nicht im Wertsinne) des instinktiven Verhaltens“: „Schöpferische Dissoziation, nicht Assoziation oder ,Synthese“ (Wundt) ist also der Grundvorgang der psychischen Entwicklung. Und dasselbe gilt auch physiologisch“ (Scheler 1995, 22f.). ${ }^{1}$ Habe die Dissoziation von Instinkten erst einmal Assoziation ermöglicht, ermögliche die Degenerierung assoziativer Systeme nicht nur eine Reduktion der Komplexität, sondern neue Assoziationsbildungen, so Edelman in der aktuellen Diskussion: „Degeneracy in brain circuits leads almost inevitably to association, a key property required for memory and learning. This associative property occurs because of the overlap of different degenerate circuits leading to a similar output.“ (Edelman 2006, 34). Die Assoziation setzt hier Schaltkreise auch schon voraus, statt solche erst auszubilden. Zudem werde die Assoziation gerade dadurch ermöglicht, dass die neuronalen Zirkel degenerieren, statt die Verbindung sensorischer mit motorischen Organen zu generieren.

Dass das physische Korrelat für Selbstbewusstsein auch eine Selbstreferenz aufweisen könnte, ist in Schelers und Plessners Rahmentheorie keine Überraschung. Plessner hat davon gesprochen, dass man bereits in der zentrischen Positionalität von Tieren das Gehirn als Organ der Pause von der Verknüpfung der Sensorik und Motorik begreifen müsse. Erst wenn die interne Selbstreferenz des Gehirnes es ermögliche, davon Abstand zu nehmen, dass der Organismus auf sensorische Eingänge sofort durch motorische Antworten reagiere, könne man ein Korrelat für intelligentes Verhalten erwarten.

Dass die Vergangenheit nicht so, wie sie war, sondern in gegeneinander verschiebbaren Fragmenten und Elementen die historische Reaktionsbasis bildet, hat seinen inneren Grund in der Unterbrechung oder Pause, welche das im Selbstvollzug vermittelte Sein eines bewussten Lebewesens ausmacht. Der Selbstvollzug, auf dem die Möglichkeit des Be-

1 Scheler widerspricht ausdrücklich der bis heute üblichen, damals auch von Karl Bühler vertretenen Auffassung, dass Intelligenz erst zum assoziativen Leben der höchsten Säugetiere hinzutrete (Scheler 1995, 23). Eric Kandel hat dann in der Tat solche Lernmechanismen bereits bei wirbellosen Tieren aufgedeckt, die über eine epigenetische Regulation der Gen-Expression auf molekularbiologischer Ebene laufen können (Kandel 2006, 205f., 225). 
wusstseins beruht, wirkt, könnte man auch sagen - eben weil er die innere Pause oder Unterbrechung zwischen dem Lebendigen und ihm selber ist - wie ein Sieb, dessen Poren die hindurchtretende Masse zerstäubt, indem sie Vergangenheit wird. (Plessner 1975, 284)

Die Ermöglichungsrichtung bewussten Verhaltens komme spontan aus der offenen Zukunft, nicht aus einer abgeschlossenen Vergangenheit. Die heutigen Modelle von der selbstreferentiellen Funktionsweise des Gehirns durch zeitliche Synchronisation passen in den hypothetischen Rahmen von Scheler und Plessner, ohne dass natürlich beide den heutigen neurobiologischen Forschungsstand hätten voraussehen können.

Außer dieser paradigmatischen Umorientierung in der Neurobiologie auf die Selbstreferenz in der Funktionsweise des Gehirnes war sicherlich die Entdeckung der Spiegelneuronen bei Makaken durch Rizzolatti u. a. ein wichtiger Fortschritt im empirischen Sinne. Sie passen aber nicht in die gerade erörterte Funktionsdynamik von Metarepräsentationen, sondern führen in die Frage nach den spezifischen Funktionen bestimmter Repräsentationsarten zurück, für die es offenbar schon besondere genetische Anlagen gibt. Inzwischen nimmt man an, dass solche Spiegelneuronen nicht nur bei Affen, sondern auch Menschen vorkommen. Ihre Aktivität korreliert nicht nur mit der Wahrnehmung von Handbewegungen durch Artgenossen oder körperlich ähnlich gebaute Lebewesen, sondern auch mit Gesichtsausdrücken und Lauten solcher Lebewesen, also unter Affen oder unter Primaten auch zwischen den Arten. Von besonderem Interesse sind dabei Spiegelneuronen in dem Feld F5c im Großhirn von Menschenaffen und im Brodmann-Areal 44 bei Menschen, das für die Sprachbearbeitung bedeutsam ist. Die Spezifik dieser Neuronen besteht darin, dass sie im Falle der Beobachtung anderer ähnlicher Lebewesen selbst ein Aktivitätsmuster generieren, das sie ansonsten nur aufweisen, wenn der eigene Körper, zu dem sie gehören, dieses Verhalten ausführt, also z. B. das Ergreifen einer Frucht mit der Hand, ein Gähnen, mit dem sich das andere Lebewesen zurücklehnt, oder ein Spielgesicht, ein Jagdgesicht, mit dem das andere Lebewesen zur Fortbewegung ansetzt, ein Ausstrecken der Zunge, auf das Babys ebenso antworten (siehe zusammenfassend Iacobini 2008). Offenbar liegen Spiegelneuronen in einer Rückkopplungsschleife, die eine bestimmte Verbindung zwischen Sensorik und Motorik in Bereitschaft versetzt. Ein Lebewesen führt eine bestimmte Verhaltenseinheit aus und das andere, ähnlich gebaute Lebewesen nimmt das erstere Lebewesen so wahr, dass es in die Ausführung der gleichen Verhaltenseinheit hineingerät. Erwachsene Lebewesen können die Ausführung des gleichen Verhaltens korrigieren, stoppen oder verkürzen, aber sie geraten erst einmal spontan in die Bereitschaft zur Ausführung der gleichen Verhaltenseinheit, die das erste Lebewesen bereits ausführt. 
Max Scheler hat die Phänomene, die man heute mit den Spiegelneuronen als Korrelat verknüpft, die Phänomene der Gefühlsansteckung und des Einsfühlens im vitalen Verhalten genannt. Er hat aber darauf bestanden, dass man diese vitalen Verhaltensphänomene der Ansteckung und des Einsfühlens nicht verwechselt mit den spezifisch menschlichen Mitgefühlen, die nicht auf der vitalen Ebene, sondern auf der personalen Ebene des Verhaltens liegen (Scheler 1985, 105-149). In Helmuth Plessners Terminologie stellen die Spiegelneuronen neuronale Korrelate für das dar, was er das Mitmachen im Unterschied zum Nachmachen und zum Nachahmen genannt hat. Bevor ich darauf zurückkomme, lässt sich aus der Sicht dieser Philosophischen Anthropologien Folgendes sagen: Die Entdeckung der Spiegelneuronen bedeutet die Entdeckung von neuronalen Korrelaten für das Mitmachen bzw. die Gefühlsansteckung durch und das vitale Einsfühlen in bestimmtes Verhalten, aber immer hier und jetzt. Dies ist eine große Leistung, aber sie wird missverstanden, wenn man sie für die Erklärung intelligenten und geistigen Verhaltens durch Nachahmung hält. Nachahmen ist mehr als nachmachen und mitmachen. Dieses Problem ist in der neueren Verhaltensforschung wieder aufgetaucht. Leider nehmen die Überinterpretationen der Spiegelneuronen in der Hirnforschung nicht die Differenzierungen in der Verhaltensforschung zur Kenntnis.

\subsection{Zur Verhaltensforschung zwischen Frans de Waals Aufwertung der emotionalen Leistungen von Menschenaffen und Michael Tomasellos Begrenzung der symbolisch kooperativen Kommunikation auf Menschen}

De Waals Forschungsprogramm betont die Kontinuitäten zwischen den Menschenaffen (nicht den anderen Affen) und dem Menschen. Für ihn gibt es insbesondere eine moralische Kontinuität zwischen beiden, wobei diese Kontinuität von unten her bei zwei Emotionen ansetzt, der Empathie und der Reziprozität. Er verortet seine eigene Konzeption in dem Zusammenhang der alten Diskussion seit Theodor Lipps über Einfühlung und seit Scheler über die Vielfalt der Mitgefühle (vgl. zu dieser Diskussion Schloßberger 2005). De Waal stellt auf der untersten gemeinsamen Ebene, emotionale Übertragung genannt, die Parallele $\mathrm{zu}$ Schelers Gefühlsansteckung und zu den Spiegelneuronen im Sinne von Gallese her. Dabei handele es sich um eine automatische emotionale Beeinflussung, die kaum oder später bewusst werde und sich von der kognitiven Empathie unterscheide (de Waal 2008, 57-59). Neben dieser emotionalen Empathie gebe es auch die emotionale Erwartung von Reziprozität bereits unter den Men- 
schenaffen. Die Reziprozität zwischen Geben und Nehmen werde nicht in ein und derselben Situation erwartet, sondern langfristig über viele Situationen hinweg, seien es Wochen und Monate oder sogar Jahre. Diese Basisebene aus emotionaler Empathie und Reziprozität werde auch schon unter Menschenaffen durch eine zweite Ebene des sozialen Drucks in der jeweiligen Gruppe erweitert. Sie schließe an Verwandtschaftsbeziehungen in der Generationenfolge, Ranking-Kämpfe und Bündnisbeziehungen gegenüber Dritten an, insbesondere wenn es sich bei diesen Dritten um andere Gruppen handelt. Der soziale Druck sei aber unter Menschenaffen nicht so stabil wie unter Menschen, die für Belohnung, Strafe und Reputationsaufbau spezielle Institutionen gründen. Die dritte Ebene der Moral, die auf den beiden ersten Ebenen aufbaue, bestehe aus der Internalisierung der Bedürfnisse und Ziele anderer, so dass letztere in das moralische Urteil eingehen, das letztlich selbstreflexiv sei. Für diese moralische Vernunft im engeren Sinne fänden sich keine Ähnlichkeiten zu anderen Menschenaffen (ebd., 187-195). Zu ihr würde die Ambivalenz der spezifisch menschlichen Mitgefühle gehören, wie sie Scheler beschrieben hat. In der kognitiven Empathie (Ebene 3), nicht in der emotionalen Empathie (Ebene 1), kann man mit einer Person, die Schmerzen hat, mitfühlen, ohne dass man jetzt dieselben Schmerzen hat, womöglich sogar ohne dass man diese Schmerzensart je hatte. Dies betrifft auch die Mitfreude, die Scheler im engeren Sinne Sympathie nennt.

De Waals Forschungsprogramm ist seit Jahrzehnten stark kritisiert worden von Verhaltensforschern, die seine Beobachtungen nicht reproduzieren konnten. De Waals Beobachtungen beruhen zu einem großen Teil auf teilnehmender Beobachtung an dem Leben von Menschenaffengruppen in freier Wildbahn. Die Kritik lautete häufig: Darin steckt zu viel unkontrollierter Anthropomorphismus. Das raffinierteste Forschungsprogramm zur methodischen Kontrolle des Versuchsdesigns mit Menschenaffen, die in Zoogruppen leben, stammt von Michael Tomasello und seinen Mitarbeitern am Max Planck-Institut für Evolutionäre Anthropologie in Leipzig. Er stellt seit Jahrzehnten Untersuchungen an, die nicht nur Menschenaffen untereinander betreffen, sondern auch den Vergleich mit der Humanontogenese von Menschenkindern. Für ihn gibt es ebenso wie für de Waal und die Philosophische Anthropologie viele Gemeinsamkeiten zwischen Menschenaffen und Menschen, aber er beharrt auch auf einem entscheidenden Unterschied: Menschenkinder folgten schon ab dem 9. bis 12. Monat symbolischen Gesten, die ihre Bezugspersonen ausüben, um sie mit ihnen zu teilen. Sie seien bereits biologisch dafür adaptiert, mit ihren Bezugspersonen ihre Intentionalität gegenüber Gegenständen und Lebewesen teilen zu wollen, kurz: shared intentionality genannt. Dadurch wachsen sie früh in symbolische Dreiecke hinein. Sie identifizieren sich erst mit der Bezugsperson (dyadische 
Protokonversation), um dann von dieser Bezugsperson die Handlungen und sprachlichen Benennungen von dritten Objekten oder dritten Lebewesen zu erlernen (Tomasello 2003, 26-29). Dieses anders, nämlich am Zeigen und Blicken orientierte Potential von Menschenkindern entfalte sich in ihrem dritten Lebensjahr zu einem Spracherwerb, mit dem die Menschenaffen nicht mehr Schritt halten können. Akkulturierte, also unter Menschen aufgewachsene Menschenaffen könnten zweifellos die menschliche Sprache auf Umwegen (YerkesTastatur) erlernen, aber sie verwendeten sie zur Bezeichnung ihrer Wahrnehmungen und Vorstellungen. Sie verstünden nicht syntaktisch verallgemeinerte Marker der Sprache, die sich von den eigenen Sinneserlebnissen lösen. Menschenaffen nähmen nicht wie Menschenkinder an Prozessen der Grammatikalisierung ihres Verhaltens teil. Sie kämen nicht im Diskurs und der Narration einer Sprechergemeinschaft an, wie das bei Menschenkindern im 4. bis 6. Lebensjahr der Fall ist (Tomasello 2003, 268-279). Dazu gehört auch die Entdeckung und Erfindung von phantastischen, rein fiktionalen Wesen, denen man nie in der sinnlichen Realität begegnen kann, wohl aber in symbolischen Übertragungen. Man könnte diese Einsichten wie folgt zusammenfassen: Menschenaffen können an keine Gespenster glauben, Menschen aber sehr wohl. Povinelli (2000) formuliert deutlich: Menschenaffen seien die wahren Positivisten. Sie könnten ihr Verhalten nicht auf Sprache im Sinne des Unterschieds von Syntax, Semantik und Pragmatik umstellen. Sie verstünden diesen für uns so selbstverständlichen Unterschied einfach nicht.

Es ist sehr schwer, den Unterschied zwischen Menschenaffen und Menschen wirklich $\mathrm{zu}$ begreifen und auch empirisch überzeugend nachzuweisen. Tomasello hat dafür nicht nur die Unterschiede im Sprach- und Symbolerwerb hervorgehoben, sondern auch mit zwei weiteren Differenzen gearbeitet. Er unterscheidet zwischen „Mimicry“ und „Imitation“ (Tomasello 2002, 37). Mimicry bedeute, dass die Jungtiere besonders daran angepasst seien, das tatsächliche Verhalten ihrer Artgenossen zu reproduzieren. Dabei verstünden aber die Jungen nicht, welche instrumentelle Wirksamkeit ihre Reproduktion in der Umgebung hat, d. h. sie können nicht zwischen Ziel und Mittel unterscheiden, sondern machen einfach nach. Sie übertrügen auch nicht das reproduzierte Verhalten, das aus einer engen Verhaltensart stammt, z. B. aus dem Termitenangeln mit der Hilfe von Stöckchen, auf eine andere Verhaltensart. Dies alles sei aber bei der Imitation der Fall. In der Imitation reproduziere das Menschenkind das Verhalten des Erwachsenen, um dasselbe Ziel wie der Erwachsene zu erreichen, $d$. h. es verstehe, dass dasselbe Ziel auch durch ein anderes Mittel erreicht werden könne. Es verstehe auch, dass die Ziel-Mittel-Unterscheidung auf andere Verhaltensarten übertragen werden kann. Während menschliche Erwachsene 
bewusst eine Lehrfunktion gegenüber den Kindern ausüben, lernten die Menschenaffenkinder gleichsam nebenbei durch Mimicry, ohne den Unterschied zwischen Ziel und Mittel und ohne die Transitivität des Handelns zu erlernen. Das Handeln bleibe auf eine Verhaltensart, z. B. Futterbeschaffung oder Fortpflanzung oder Kampf um einen Rankingplatz, beschränkt.

Die andere Unterscheidung, mit der Tomasello ebenfalls experimentiert, ist die zwischen geteilten Intentionen (shared intentionality) erster und zweiter Ordnung (Tomasello 2003). Er akzeptiert, dass große Menschenaffen in einer bestimmten Verhaltensart geteilte Intentionen verstehen. Sie folgen den Blickrichtungen ihrer Artgenossen und Menschen auf externe Handlungsziele, z. B. Früchte oder anderes Futter. Sie verstehen auch den Unterschied dazwischen, ob andere etwas gesehen haben können oder nicht gesehen haben können, z. B. verstecken, oder ob andere nur bei der Ausführung ihres Zieles sensomotorisch verunglücken oder täuschen. Aber dies bleibe immer an Situationen der Wahrnehmung oder Nicht-Wahrnehmung und der Vorstellung oder Nicht-Vorstellung des Wahrgenommenen gebunden. Es bleibe auch immer beschränkt auf einen bestimmten Verhaltenskontext, z. B. der gemeinsamen Jagd außen stehender Dritter, z. B. kleiner Affen oder Artgenossen aus einer anderen Gruppe und einem anderen Territorium zur eigenen Verteidigung oder dem gemeinsamen Angriff. Es gebe keine symbolischen Übertragungen auf andere als dem Trieb vorgegebene Verhaltensarten. Die Kooperation einer Schimpansengruppe während der Jagd sei das beste Beispiel für eine shared intentionality erster Ordnung, aber sie werde nicht auf andere Verhaltensweisen übertragen. Den Teilnehmern werde auch nicht bewusst, welche Teilfunktion sie in einem kollektiven Handlungsplan ausüben. Das Letztere erfordere eine shared intentionality zweiten Grades, d. h. eine kollektive Intentionalität (we-intentionality), die es in Gruppen von Menschenaffen nicht gebe (Tomasello/Rakoszy 2003; Tomasello 2005). Menschen könnten dagegen kooperieren, weil sie kommunizieren. Sie können auch kommunizieren, um neue Kooperationen auszuhandeln, d. h. ihre Kommunikation symbolisch auf neue Kooperation übertragen. Dies können Menschenaffen nicht. Sie bilden ebenso keine stabilen Rollen und Institutionen aus, die die aktuelle Existenz ihrer Generation und Gruppe überdauern. Sie kennen akute Trauer und Tröstung, wenn jemand aus ihrer Gruppe stirbt, aber es gibt keine Symbole, die an die Verstorbenen in der Generationenfolge wie bei Menschen erinnern. 


\subsection{Philosophische Anthropologie als Rahmen für den Zusammenhang zwischen der Hirn- und Verhaltensforschung}

Plessner hat in seinen Stufen des Organischen (Plessner 1975) die Ermöglichungsstrukturen des personalen Lebens freigelegt. Er hat rekonstruiert, welche lebensweltlichen Präsuppositionen die Biowissenschaften in Anspruch nehmen, um Leben verstehen und erklären zu können. In einem ersten Schritt entwickelt er die Hypothese, dass sich lebendige Dinge von anorganischen Dingen dadurch unterscheiden, dass sie ihre eigene Grenze realisieren. Dadurch werden sie nicht nur von außen begrenzt, sondern vollziehen sie die Abgrenzung nach außen und die Öffnung nach außen. Sie positionieren sich in ihrem Verhalten nach außen und gehen so in sich zurück. Sie organisieren sich in ihrem Inneren und positionieren sich in der ihnen äußeren Umgebung. Damit entsteht die Frage, wie ihre Organisationsform (nach innen und von innen) mit ihrer Positionalitätsform (nach außen und von außen) zusammenhängt. Von Uexküll hatte in seiner theoretischen Biologie eine Korrelation zwischen dem Bauplan eines Organismus und seiner Umwelt entworfen. Plessner hat diese Idee der Passung zwischen Organisationsform und Positionalitätsform weiter durchgeführt. Die Lebewesen haben je nach ihrer Organisationsform verschiedene Umwelten. Die offene Organisationsform von Pflanzen mache sie zu einem unselbständigen Bestandteil ihrer Umgebung, der abhängig sei von bestimmten Medien (Wasser, Sonnenenergie etc.). Die geschlossene Organisationsform von Tieren mache sie zu einem selbständigen Bestandteil ihrer Umwelt. Die Schliessung der Organisationsform durch ein Nervensystem könne bei niederen Tieren dezentral durch verschiedene Nervenzentren für verschiedene Verhaltensarten erfolgen. Oder diese Schließung könne zentral durch ein Gehirn geschehen, z. B. das Säugergehirn, das Korrelate für verschiedene Verhaltensarten integriert. Die dezentrale oder zentrale Schließung der Organisationsform führe zu verschiedenen Umwelten für diese Tiere. Bei dezentraler Schließung reagieren die Tiere auf komplexe Qualitäten, bei zentraler Schließung auf Dinge, die verschiedene Qualitäten vereinen. Der zentrischen Organisationsform entspricht im Verhalten eine zentrische Positionalitätsform (siehe zu diesen Schritten oben 1.4. bis 1.6.).

Interessant ist das 6. Kapitel der Stufen, weil es die Schimpansenversuche von Wolfgang Köhler auswertet, der gezeigt hatte, dass Schimpansen eine praktische Intelligenz entwickeln. Für Plessner fiel in diesen Versuchen zweierlei auf: Schimpansen konnten verschiedene Dinge als Vermittlungen in die Handlungen einbauen, die sie ausführen mussten, um schließlich ihre Triebe zu 
befriedigen. Die intelligente Leistung variierte aber sehr stark von Individuum zu Individuum, was mit den verschiedenen Gedächtniskapazitäten zusammenhing. Ein gutes Gedächtnis emanzipierte von großem Aufwand in der Wahrnehmung jetzt und hier zugunsten des schnellen Vergleichs mit früheren Situationen, um die neue Situation zu meistern. Noch auffälliger war aber für Plessner dies: Je länger und schwieriger die Umwege bei der intelligenten Problemlösung wurden, desto schneller erlahmte das Interesse der Schimpansen. Ihre Intelligenz blieb als Mittel in ihre überschaubare Triebbefriedigung eingebaut, sie konnte sich nicht davon lösen. Ihnen fehlte, wie Plessner schrieb, der „Sinn für das Negative“ (ebd., 270). Sie benutzten keinen symbolischen Kontrast des ihnen Gegebenen zu einem leeren Raum und zu einer leeren Zeit. Für Schimpansen gibt es nicht das Abwesende, das außer im Symbol nie kommt, z. B. eine Idee, sondern nur ein Abwesendes, das hier und jetzt nicht mehr oder noch nicht wieder da ist. Sie gehen zwar mit Dingen um, aber nicht in einem für sie leeren Raum und in einer für sie leeren Zeit, sondern immer ihrem Trieb gemäß getönt.

In dem berühmten Schlusskapitel des Buchs führt Plessner die exzentrische Positionalität ein, um die Ermöglichungsstrukturen personalen Lebens zu rekonstruieren. Wieso können wir Menschen, darunter Biologen und Philosophen, einerseits die Gemeinsamkeiten mit Schimpansen, andererseits aber ihre Verhaltensweisen auch als wirklich andere verstehen? Intelligente Leistungen im Rahmen von Triebbefriedigungen kennen wir alle auch. Sie gehören zu der getönten Konzentrik von Leibern, die sich zentrisch positionieren. Aber darauf lassen sich personale Verhaltensweisen nicht reduzieren. Wenn Biologen und Philosophen so viele verschiedene Organisations- und Positionalitätsformen unterscheiden können, dann nehmen sie an diesen Formen nicht nur teil, dann haben sie auch Abstand zu diesen. Erst durch diesen Abstand können sie solche Formen als solche Formen von anderen Erkenntnisgegenständen unterscheiden lernen. Diese Distanz nennt Plessner die Exzentrik im Unterschied zur Konzentrik des Verhaltens von Primaten. Personale Lebewesen stehen nicht nur frontal den Dingen und anderen Lebewesen in ihrer Umwelt gegenüber, sondern stehen auch neben diesen Relationen zwischen ihrem Organismus und ihrer Umwelt in einer Welt. Personale Verhaltensweisen sind triadisch und damit symbolisch, wie es Tomasello in seinem Forschungsprogramm bestätigt hat. Aus dieser exzentrischen Welt von Personen heraus lassen sich die verschiedenen Umwelten und Organisationsformen unterscheiden, weil dieser Weltrahmen einen Abstand davon gewährt. An die Stelle der dyadischen Relation zwischen Organismus und Umwelt treten die triadischen Relationen in der Außen-, Innen- und Mitwelt. Die Mitwelt besteht aus den Relationen der Personen unter- 
einander, also Interpersonalität, und der Relation solcher Personalität zu Körpern und Leibern. Plessner entwickelt diese Spezifik der Mitwelt in anderen Büchern durch seine Theorie des Rollenspiels weiter, wodurch die Sozial- an die Naturphilosophie anschließt.

Personale Lebewesen müssen sich die Umwelt, die ihre zentrische Organisationsform braucht, erst künstlich, vermittelt und utopisch schaffen, also auf dem Wege soziokultureller Weltrahmen. Das Begriffsgefüge der exzentrischen Positionalität enthält also das Problem, wie eine zentrische Organisationsform und eine exzentrische Positionalitätsform zueinander passen können. Eine zentrische Organisationsform braucht eine zentrische Positionalitätsform, $d . h$. die Interaktion zwischen dem Organismus und der Umwelt. Dieser Vordergrund scheint von einem Hintergrund her, aus einer Welt und damit auf Abstand vollzogen zu werden. Diese naturphilosophische Problemstellung wird unter drei Aspekten entfaltet: Wie kann ermöglicht werden, dass Künstlichkeit in der Aussenwelt natürlich wird? Wie ist es in einer Innenwelt möglich, dass Unmittelbarkeit vermittelt wird? Und inwiefern kann in einer Mitwelt Utopisches wirklich werden? (ebd., 309f., 321f., 341f.). Diese naturphilosophische Fundierung bedarf der sozial- und kulturphilosophischen Ergänzung im Medium der Geschichte des personalen Lebens (ebd., 32-36; dazu im vorliegenden Band zweiter und dritter Teil).

Die Hirnforschung ist mit dem Zentralorgan der zentrischen Organisationsform, eben dem Gehirn, beschäftigt, das als neurophysisches Korrelat für den Vollzug der leiblichen Konzentrik fungiert. Wenn schon die zentrische Positionalität die o. g. Pause (Unterbrechung) der Verbindung zwischen Sensorik und Motorik beinhaltet, dann lässt die exzentrische Positionalität eine Steigerung davon erwarten, nämlich die Pause von dieser Pause, eben die soziokulturelle Unterbrechung von der biosozialen Unterbrechung. Die Verhaltensforschung untersucht verschiedene Verhaltensweisen, a-zentrische, dezentral und zentral geschlossene, konzentrische und exzentrische Verhaltensweisen, die in den entsprechenden Positionalitätsformen vorkommen können. In diesem naturund sozialphilosophischen Rahmen der Philosophischen Anthropologie können die Hirn- und Verhaltensforschung in einen Zusammenhang gebracht werden, was beide nicht systematisch von sich aus tun und ich daher in meinen Büchern Zwischen Lachen und Weinen. Bd. 1: Das Spektrum menschlicher Phänomene (Krüger 1999, 3. Kap.) und Gehirn, Verhalten und Zeit (Krüger 2010) vorgeschlagen habe. Schauen wir uns diesen Zusammenhang zum Abschluss anhand einer begrifflichen Unterscheidung genauer an, deren Zusammenhang mit anderen kategorialen Unterscheidungen hier vorausgesetzt werden muss (Krüger 2010, 1. Kap.). 


\subsection{Der Unterschied und Zusammenhang zwischen Mitmachen, Nachmachen und Nachahmung}

Das Mitmachen bezieht sich auf mindestens zwei Lebewesen einer ähnlichen Organismusart, die hier und jetzt in einer für einander präsenten Wahrnehmungssituation aufeinander treffen. Es findet im Rahmen des Zusammenspiels von zentrischer Organisationsform und zentrischer Positionalitätsform statt, d. h. in zentrischen „Mitverhältnissen“ (Plessner 1975, 306-308). In der Situation des Mitmachens kommt es zur Überlagerung zweier konzentrischer Verhaltenskreise. Das eine Lebewesen löst in dieser Überlagerung in dem anderen Lebewesen eine ähnliche Verhaltenseinheit aus. Wie ist dies möglich, ohne dafür eine bewusste Einfühlung oder einen bewussten Analogieschluss des zweiten Lebewesens annehmen zu müssen? Sie stecken einander in einem gefühlten Impuls an, indem das zweite Lebewesen in ähnliche Bewegungen wie das erste Lebewesen gerät. Die Ähnlichkeit des Verhaltens ist nicht verwunderlich, weil sie in der Ähnlichkeit der Organismusart vorausgesetzt wird. Die Ähnlichkeit besteht für uns, sie wird nicht von den beteiligten Lebewesen bewusst ermittelt. Letztere folgen ihren Instinkten, Trieben und Prägungen, werden aber in der Überlagerung ihrer leiblichen Konzentriken gegenseitig zu Impulsgebern ihres Verhaltens.

Was Plessner das vital leibliche Zusammenspiel zweier Lebewesen nennt, betrifft gerade nicht den je eigenen Körper im Gegensatz zu anderen Körpern, sondern die spontane Überlagerung beider Konzentriken, die keinem von beiden allein gehört, sondern von beiden geteilt wird. Die leibliche Dimension beider liegt vor der Unterscheidung und Zurechnung nach Eigenem und Anderem für das betroffene Lebewesen. Dies kann man in den Spielen der Säuger, z. B. von Hunden oder Delphinen, deren Körper auf der Kreislinie um den von ihnen geteilten Leib in der Kreismitte herumtanzen, sehr gut sehen. Die sogenannten Spiegelneuronen stellen inzwischen für Affen die Entdeckung eines neurophysischen Korrelats für das Mitmachen dar. Wenn sie so wie oben charakterisiert, d.h. als Bereitschaftspotential, fungieren, dann bestätigt diese Entdeckung Plessners phänomenologische Einsicht, dass im Mitmachen das betroffene Lebewesen nicht zwischen eigenem und anderem Verhalten als solchen unterscheiden kann.

Ich halte aber die Bezeichnung dieser Neuronen als Spiegel-Neuronen für ein Missverständnis, das zu großen metaphorischen Übertreibungen geführt hat (so bei Iacobini 2008). Sie erscheinen so als ein sowohl leiblicher als auch ein geistiger Spiegel, der in der modernen westlichen Kultur auch zu einem Instrument und Bild (Medium) für die Reflexion des Bewusstseins auf Bewusstsein 
geworden ist. Die zentrischen Lebewesen, die untereinander mitmachen, teilen indessen nicht unser Selbstverständnis. Bei ihnen handelt es sich im Falle der sogenannten Spiegelneuronen um einen angeborenen, nur auszuwachsenden, unbewusst anlaufenden Mechanismus der Auslösung des gleichen Bereitschaftspotentials (siehe oben). Es geht noch nicht einmal um die Frage des leiblichen Selbstbewusstseins, das man durch Versuche mit tatsächlichen Spiegeln (nicht deren Metaphern) an Walen, Elefanten, Affen etc. erfolgreich getestet hat. Zur leiblichen Konzentrik von Verhalten gehört natürlich der Vollzug des leiblichen Selbst, das sich in der Überlagerung verschiedener Konzentriken als eigenes abheben kann. Aber dieses leibliche Selbstbewusstsein darf man nicht mit einem geistigen Selbstbewusstsein verwechseln. Plessner hat diesen Unterschied aus der Differenz zwischen leiblichem und reinem Ich entwickelt (siehe im vorliegenden Band drittes Kapitel).

Das Nachmachen bezieht sich bei Plessner auf zentrische Lebewesen, die sich - in Abhängigkeit von ihren Gedächtniskapazitäten - erneut, mithin durch die jetzige Situation modifiziert vorstellen, welche Wahrnehmungen sie auf dem Wege einer früheren Triebbefriedigung hatten. Das „Nach“ im Nach-Machen ist also zeitlich gemeint und unterstellt, dass der gleiche Trieb künftig erneut befriedigt werden wird, wodurch es zu der Erinnerung an frühere Triebbefriedigungen kommt. Das Nachmachen variiert je nach neuer Situation das Mitmachen im zeitlichen Nacheinander. Es stellt einerseits eine Wiederholung, andererseits eine Abweichung davon dar. Die Wiederholung kann zu Gesten führen, die nur für die beteiligten Lebewesen eine Verhaltensbedeutung haben, d. h. die nicht symbolisch verallgemeinert werden können. Dyadische Gesten können Einladung, Gleichgültigkeit, Sympathie und Antipathie, Unterwerfung oder Dominanz, Gleichberechtigung im Verhalten signalisieren. An diese gestischen Erwiderungen, die Tomasello ausführlich beschreibt, können sich intelligente Leistungen in der Verfolgung von Triebbefriedigungen anschließen, wie sie für Menschenaffen seit langem bekannt sind, z. B. auch in gemeinsamer Jagd auf außen stehende Dritte oder in der Schmiedung von Allianzen gegen Dritte in der eigenen Gruppe. Ähnlich wie schon Scheler und Plessner für das Verstehen solcher Phänomene unter ihren Teilnehmern keine spezifisch geistige Verhaltensdimension veranschlagt haben, halten auch Tomasello und Povinelli hierfür keine Wir-Intentionalität, $d$. h. keine geteilte kollektive Intention über geteilte Intentionen, für nötig. Solche Verhaltensphänomene ergeben sich für die betroffenen Lebewesen aus der leiblichen Überlagerung der Konzentriken, in der sie alle intelligent ihrer Triebdynamik folgen und untereinander eine gestische Vertrautheit aus früheren Erfahrungen des Mitmachens und des Nachmachens entwickeln. Diese Erfahrungen beziehen sich auf eine bestimmte Verhal- 
tensart, sie werden von niemandem der beteiligten Lebewesen symbolisch auf andere Verhaltensarten übertragen. Nur wir Menschen können uns eine Jagd nicht anders vorstellen als dadurch, dass es eine Praxis des Einübens, der Kommunikation und der arbeitsteiligen Rollenverteilung gibt. Das leibliche Selbstbewusstsein kann aus dem Nachmachen in sozialen Mitverhältnissen hervorgehen.

Was Plessner schließlich Nachahmung nennt, bezieht sich auf die Beantwortung zweier unterschiedlicher Fragen, wenngleich diese zusammenhängen: Was, d. h. welcher Sachverhalt, wird nachgeahmt? Und: Wer, d. h. welche Person, wird nachgeahmt? Die Frage, ob „echte Nachahmung“ vorliegt, sollte also an dem Nachmachen den folgenden Unterschied antreffen und ausweisen können: „Jemandem etwas nach-machen ist nicht dasselbe wie jemanden nachmachen“ (Plessner 1982h, 397). Es macht einen Unterschied, ob man bei der Fahrradreparatur einen Sachverhalt nachahmt, z. B. ein Rad auswechselt, oder ob man die Person, die versucht, ein Fahrrad zu reparieren, nachahmt. In beiden Fällen, sowohl der Darstellung von Sachverhalten als auch der Darstellung von Personen, gibt es Muster oder Bilder, an denen man sich auf eine symbolisch allgemeine Weise orientiert. Solche Darstellung wird symbolisch erlernt und schwebt dann in der Imagination vor, an deren Vorstellungen man seine wahrnehmbaren Verhaltenseinheiten ausführt. Dafür nimmt man sinnlich Abwesendes als symbolischen Kontrast in Anspruch, wie man es in einer Mitwelt zwischen Personen und einem selbst erlernt hat. Plessner nennt den Modus der Darstellung von Sachverhalten und Personen den bewusst gewordenen „kategorischen Konjunktiv“ (Plessner 1983b). Beste Beispiele für die Darstellung von Sachverhalten sind Wissenschaft und Technik, für die Darstellung von Personen Schauspiele und andere Künste. Aber diese Ausdifferenzierungen und Spezialisierungen setzen voraus, dass es beide Nachahmungen oder, gleichbedeutend, Darstellungen in der alltäglichen Lebenswelt von Personen gibt.

Plessner würdigt in seinem Spätwerk nach dem zweiten Weltkrieg die phänomenologischen Leistungen von Jean-Paul Sartre und Maurice Merleau-Ponty im Anschluss an Scheler, um der Ausarbeitung der Frage, was Nachahmung über Mit- und Nachmachen hinausgehend auszeichnet, näher zu kommen.

Wir leugnen den Einfluss von Sympathie und Antipathie auf die Imitation ebenso wenig als das Phänomen der Intersubjektivität der ausdruckshaften Züge und der in ein Verhalten, Benehmen, Gebaren eingehenden Bewegungen. Aber die Möglichkeit der Nachahmung verlangt mehr, zumal wenn sie nicht in affektiver Miterregung, sondern als bewusste Handlung zum Zweck der Nachbildung auftritt. Hier muss eine lokalisierte Übertragung der gesehenen Gestalt in die mir verfügbare Zone meines Aussehens stattfinden können, die mir als solche primär nicht visuell gegeben ist. (Plessner 1982h, 393f.) 
Frage man sich, wer oder was denn die Entsprechung zwischen dem fremden Vorbild und den eigenen Bewegungen regele, bestehe das Nächstliegende in dem

[...] Blick, der dem Blick des Anderen begegnet. In ihm haben wir das elementare Phänomen der Reziprozität zwischen mir und dem Anderen. Sobald mein Blick das fremde Auge trifft, sehe ich mich erblickt, angeblickt - und nicht nur (etwa in der Art des Augenarztes) das Auge. Der Andere sieht nicht nur aus, sondern - mich an und steht damit in der Position des Vis-à-vis als derjenige, mit dem ich den Platz tauschen kann. In dieser Vertauschbarkeit des Blickpunkts, die mir sein Blick bezeugt, ist er ein Anderer, bin ich für ihn ein Anderer. [...] Indem die Durchlässigkeit des erblickten Blicks mein Blicken an seine Augen fixiert, ist mir zugleich die Symmetrieebene gesichert, um die mein mir selbst nicht sichtbares Gesicht sich ordnet. Am ,Leitfaden' des begegnenden Blicks kann daher die Entdeckung der Reziprozität des Körperschemas erfolgen, dessen Ausbildung mit der Ausbildung und Beherrschung der Motorik vermutlich gleichen Schritt hält. Gerade weil meine Augen, ,mit‘ denen ich blicke, mir selbst unsichtbar bleiben, treten seine Augen als Blicksender und Blickfänger zu ihnen ins Wechselverhältnis. Damit ist die Abbildung seines Gesichts und schließlich seiner ganzen Gestalt auf mein Bewegungsschema möglich geworden. (Ebd., 394f.)

Plessner schließt sich dem Fortschritt in der Präzisierung der Fragestellung an, der in der Kombination der Ansätze von Sartre und Merleau-Ponty besteht:

Als exzentrisches Wesen sich selbst gegenwärtig (Sartre) und in eins damit undurchsichtig (Merleau-Ponty) verfügt der Mensch über die einander begegnenden Blicke als Leitfaden zwischen sich und dem Anderen, an dessen Stelle er stehen könnte und dessen Mienen und Gesten er daher auch mit seinem eigenen Leibe nachahmen kann. (Ebd., 396)

Gleichwohl hält Plessner diesen Fortschritt eben doch nicht für die Lösung des Problems der Nachahmung, denn noch immer wird dasjenige Spektrum im Verhältnis zwischen mir und dem Anderen vorausgesetzt, das zwischen völliger Identifikation mit ihm und völliger Ablösung von ihm schwankt.

Darum wäre es auch verkehrt, die Entdeckung der exzentrischen Stellung zu mir und dem Anderen, meinem und seinem Leib der Begegnung des Blicks allein anzuvertrauen oder gar auf sie zurückzuführen. Die Sache liegt umgekehrt. Auf Grund der Exzentrizität vermag der Mensch den Blick als Leitfaden, als Führungslinie zu sehen, die ihm [...] das Verständnis für die Reziprozität des Körperschemas, d. h. die Abbildbarkeit des fremden Leibes auf seinen Leib eröffnet und in eins damit auch wirklich sichert. (Ebd., 396)

Die exzentrische Positionalität bleibt für Plessner der offene Ermöglichungsgrund, den wir schon immer in Anspruch nehmen, um Aspekte der Nachahmung thematisieren und klären zu können. Dies gilt auch für die von Plessner selbst vorgeschlagene Theorie des Schauspielens in und mit soziokulturellen 
Rollen für Personen, den Figuren des „Doppelgängertums“ (Plessner 1982b, 453), denen hier nicht mehr nachgegangen werden kann (dazu Krüger 1999, 4.-6. Kap.; im vorliegenden Band Zweiter Teil). Das Verstehen und Erklären der Triaden zwischen Personen, Körpern und Leibern setzt mehr als diese Triaden voraus, wie sich in der geschichtlichen Änderung entsprechender Regeln für ihre Entgegensetzung und Zuordnung zeigt. Auf dieses „Mehr“ einer gegenüber der Vergangenheit offenen Zukunft zielt Plessner in seiner Freilegung der Ermöglichungsstrukturen personalen Lebens ab, sowohl in der Naturphilosophie (,exzentrische Positionalität“) als auch in der Geschichtsphilosophie (homo absconditus: der Unergründlichkeit des Menschen im Ganzen seines Wesens). Eine paradigmatisch ähnliche Rolle spielt in Tomasellos Werk der sogenannte Vogel-Blick (birds view), inzwischen im Anschluss an Thomas Nagel auch als „View from nowhere“ bezeichnet, aus dem heraus neues Lernen möglich wird. Mit dieser Metapher, in der Tomasellos Verständnis der lebendigen Natur als Ermöglichungsstruktur kulminiert, hält er sich frei von jener vollständigen Identifikation von einem selbst mit einem Anderen (im Sinne des symbolischen Interaktionismus G. H. Meads), die den Konformismus mit der je eigenen Gruppe oder Gemeinschaft im Gegensatz zu anderen Gruppen oder Gemeinschaften zur Kehrseite hat (Tomasello 2009b, 40-47, 88f., 93f.). Geht es nicht nur darum, in Triaden zu leben und sie zu erleben, sondern auch darum, sie als solche zu verstehen und mit ihnen operieren zu können, erscheint die dafür nötige Ermöglichung als ein Viertes (Krüger 2010, 155; Brandt 2012, 331).

Aber kommen wir zum Abschluss hier auf den Zusammenhang zwischen dem Mitmachen, dem Nachmachen und der Nachahmung im Sinne von Darstellung zurück: Es ist bei Plessner klar, dass es Nachahmungen nicht ohne Mitmachen und Nachmachen in der Verhaltensgenese geben kann. Beide Voraussetzungen müssen bei Nachahmungen habituell mitlaufen. Die Umkehrung ist aber sehr wohl möglich: Es gibt Lebewesen, die mitmachen und nachmachen, aber nicht nachahmen können, weil sie weder Sachverhalte noch Personen darzustellen vermögen. Mimicry ist nicht imitation (Tomasello siehe oben). Es wäre ein Anthropomorphismus, ihnen nur unser eigenes Verhalten zu unterstellen statt anzuerkennen, dass Andere anders sind und anders bleiben können.

Der kognitive Unterschied zwischen Menschenaffen und Menschen begründet bei Plessner nicht per se einen moralischen Unterschied, ebenso wenig wie bei Tomasello oder de Waal. De Waal sagt treffend, so sehr er für eine Sonderstellung der Menschenaffen kämpfe, es helfe nicht, ihnen Rechte zu verleihen, die sie dann nicht ausüben könnten und die im Notfall für Menschen, die keinen Personenstatus haben, in ein Dilemma führen (de Waal 2008, 184). Die Verantwortung für das Wohlergehen der Menschenaffen liegt bei den Men- 
schen, die dank ihres Personenstatus Menschen sein können. Sie können sich durch keinen kognitiven Unterschied, der eine große Gemeinsamkeit mit den anderen Primaten voraussetzt, aus ihrer Verantwortung für die Anderen stehlen. Hinter der vordergründigen Gleichmacherei von Menschenaffen und Menschen im Namen der Moral steht heute oft die Bequemlichkeit der Personen, die Menschenaffen ihrem eigenen Schicksal, d. h. in Wahrheit ihrem Aussterben, zu überlassen. Menschenaffen können angesichts der Vernichtung der ihnen nötigen Umwelten durch Menschen seit langem nicht mehr sich selbst helfen, weil sie kognitiv anders als Personen leben. Wenn man nicht nur die Gemeinsamkeit, sondern auch den kognitiven Unterschied zwischen Menschenaffen und Menschen eingesehen hat, kann man sich nicht mehr der Verantwortung für sie als die Anderen von uns entziehen. Man hilft ihnen dann, weil sie anders bleiben können, nicht, weil sie kognitiv vermeintlich zu einer community of equals gehörten. 